\title{
RESEARCH
}

Open Access

\section{Vitamin C improves the therapeutic potential of human amniotic epithelial cells in premature ovarian insufficiency disease}

Shunyu Hou ${ }^{1+}$, Chenyue Ding ${ }^{2+}$, Han Shen $^{3+}$, Chunfeng Qian ${ }^{2,3}$, Qinyan Zou ${ }^{2}$, Jiafeng Lu ${ }^{2}$, Boxian Huang ${ }^{1,2,3^{*}}$, Jichun $\operatorname{Tan}^{4,5^{*}}$ and Hong $\mathrm{Li}^{2,3^{*}}$

\begin{abstract}
Background: Human amniotic epithelial cell (hAEC) transplantation holds great promise in treating premature ovarian insufficiency (POI). However, some deficient biological characteristics of hAECs restrict their application.

Methods: Vitamin C (VC) was added to the culture media of hAECs for 2 weeks. Then, the proliferative ability, migration ability, pluripotency, and self-renewal of VC-treated hAECs (VC-hAECs) were determined. Next, hAECs and VC-hAECs were transplanted into the ovaries of cyclophosphamide (CTX)-induced POI model mice. The ovarian function of $\mathrm{POI}$ mice was evaluated after transplantation by counting follicle numbers and measuring the blood levels of AMH, E2, and FSH. The rescue effects of VC-hAECs and hAECs were unveiled by coculturing with CTXdamaged human ovarian granulosa cells (hGCs) and analyzing relative marker expression. Additionally, ovarian marker expression and transplant survival were detected in POI mice after transplantation to verify the beneficial effect of VC-hAECs. The cytokine profiles of VC-hAECs and hAECs were revealed by performing a cytokine array and an ELISA to show their paracrine function.

Results: Our results indicated that VC promoted the proliferation, migration, pluripotency, and self-renewal of hAECs in vitro. The most effective concentration of $\mathrm{VC}$ was $50 \mu \mathrm{g} / \mathrm{ml}$. After transplantation into the POI mouse model, VC-hAECs reversed ovarian function more powerfully than hAECs. Human granulosa cell marker expression in CTX-damaged hGCs was increased after coculture with VC-hAECs compared with hAECs. In the ovaries of the POI mice, ovarian marker expression was greater after VC-hAEC transplantation than after hAEC transplantation. VChAECs showed higher transplant survival than hAECs. Furthermore, VC-hAECs secreted more growth factors than hAECS.

(Continued on next page)
\end{abstract}

\footnotetext{
*Correspondence: huangboxiannj@163.com; tjczjh@163.com; hongliszivf@163.com

tShunyu Hou, Chenyue Ding and Han Shen contributed equally to this work.

'Department of Obstetrics and Gynecology, Affiliated Suzhou Hospital of

Nanjing Medical University, Suzhou Municipal Hospital, Suzhou 215002,

China

${ }^{4}$ Reproductive Medical Center of Gynecology and Obstetrics Department, Shengjing Hospital of China Medical University, Shenyang 110000, China

${ }^{2}$ Center of Reproduction and Genetics, Affiliated Suzhou Hospital of Nanjing Medical University, Suzhou Municipal Hospital, Suzhou 215002, China

Full list of author information is available at the end of the article
} 
(Continued from previous page)

Conclusion: Treatment with VC promoted the proliferation, migration, self-renewal, and paracrine functions of hAECs. Additionally, VC elevated the therapeutic potential of hAECs in treating POI.

Keywords: Human amniotic epithelial cell, Vitamin C, Premature ovarian insufficiency, Growth factors

\section{Introduction}

Premature ovarian insufficiency (POI), which is also referred to as premature ovarian failure (POF), is a reversible syndrome affecting the female population under the age of 40 [1]. Characterized by ovarian function cessation, POI has become one of the leading causes of infertility in China. Accessible treatments for POI, such as hormone replacement therapy and ovulation induction, are not satisfying. Therefore, recent studies have been focused on the search for alternative treatments, such as stem cell therapy.

Ovarian regeneration after stem cell therapy results from complex and unclear factors. Researchers have demonstrated that paracrine factors, including VEGF, HGF, IGF-1, and FGF2, mediate the repair of damaged ovaries [2,3]. Others have suggested that stem cell-derived exosomes and exosomal microRNAs (miR-144-5p) inhibit ovarian cell apoptosis [4-6]. Recently, several studies noted that material transfer between host and donor cells accounts for the rescue effect of stem cell therapy [7, 8]. Additionally, we cannot rule out the possibility that transplanted stem cells may integrate into host ovaries, differentiate into ovarian cells, and replace the impaired cells of the recipients.

To date, various kinds of stem cells have been investigated to treat POI, including adipose-derived mesenchymal stem cells (hADSCs) [6], fetal liver mesenchymal stem cells [9], bone marrow mesenchymal stem cells (hBMSCs) 2 [10], human umbilical cordderived mesenchymal stem cells (hUCMSCs) [11, 12], human amniotic epithelial cells (hAECs) [13, 14], human amniotic fluid stem cells [15], and human amniotic mesenchymal stem cells (hAMSCs) [16, 17]. Previous studies have indicated that human amnionderived stem cells are easier, less invasive, and more cost-effective than to obtain hADSCs and hBMSCs because amniotic tissues are discarded after delivery $[16,18]$. Moreover, hAECs exhibit low immunogenicity and no tumorigenicity, which make them an ideal candidate for regenerative medicine $[13,19]$. However, the insufficient propagation ability and deficient paracrine function of hAECs has restricted their application in reversing ovarian dysfunction $[16,18]$.

Recently, to improve the efficacy of cell-based therapy, several solutions have been proposed, such as genetic engineering [20], scaffolds [21], and delivery system optimization [22]. The safety of genetically engineered cells is concerning due to transcriptome alterations and increased tumorigenicity. Scaffolds will support transplanted cells, but their degradation and immunogenicity do not put forth a solution. Additionally, the advancement for delivery system optimization is limited.

With small molecule compounds, cell characterizations can be regulated and modified in a nonimmunogenic, temporal, and standardized way. Vitamin $C$ (VC) is known as a natural antioxidant. Studies have shown that $\mathrm{VC}$ can accelerate proliferation, promote self-renewal, and induce a pluripotent state in a variety of stem cells [23-25]. Supplementation with VC increased histone demethylase JARID1A and pluripotency markers (NANOG, SOX2, C-MYC, and KLF4) expression [24, 26-28]. $\mathrm{VC}$ has also been reported to improve proliferation, multidifferentiation potential, and extracellular matrix secretion of BMSCs [29-31]. However, the biological characterization alteration of hAECs after VC treatment has never been investigated.

For these purposes, in the present study, we modified the biological characterizations and elevated the therapeutic potential of hAECs with the small molecule, VC.

\section{Materials and methods}

\section{Isolation and culture of human amniotic epithelial cells} (hAECs)

The preparation and culture of hAECs as well as donor information were described previously [16]. In brief, human placental tissues from healthy women who were negative for HIV-I, hepatitis B, and C were collected after informed consent was signed. The chorion was washed with PBS and then separated to obtain the amniotic membrane. Then, membrane segments were dissolved in $0.25 \%$ trypsin/EDTA (Thermo Fisher Scientific, USA) for $45 \mathrm{~min}$ at $37^{\circ} \mathrm{C}$. Then, cells were seeded onto culture plates supplemented with 10\% FBS-containing DMEM (Thermo Fisher Scientific, USA) and incubated at $37^{\circ} \mathrm{C}$ in $5 \% \mathrm{CO}_{2}$. Cells were passaged or analyzed after reaching confluence.

\section{Fluorescence-activated cell sorting (FACS)}

Approximately $2 \times 10^{5}$ hAECs, VC-hAECs, hGCs, or ovarian cells were isolated and washed with DPBS. 
Then, these $2 \times 10^{5}$ cells were each added to a tube to undergo the following experiments. PEconjugated or FITC-conjugated antibodies were incubated with the cells for $30 \mathrm{~min}$ in a dark room at room temperature (Antibody information, see Supplementary Table 1). Isotype controls were used to as negative controls. Before being tested by a fluorescence-activated cell sorter (Beckman, S. Kraemer Boulevard Brea, CA, USA), cells were washed and resuspended in $100 \mu \mathrm{l}$ of PBS. Fixation and permeation buffers (BD, USA) were used before antibody incubation when intracellular proteins were detected. At least three assays were performed for each experiment.

\section{Wound healing assay}

A culture insert (Ibidi, Germany) was employed to assay the cell migration ability of hAECs and VC-hAECs. Approximately $3 \times 10^{5}$ hAECs were seeded onto the culture insert and cultured overnight. Then, a standardized wound of $500 \mu \mathrm{m}$ was made using a sterile tweezer. Afterwards, PBS, $25 \mu \mathrm{g} / \mathrm{ml} \mathrm{VC,} 50 \mu \mathrm{g} / \mathrm{ml} \mathrm{VC}$, or $100 \mu \mathrm{g} /$ $\mathrm{ml} \mathrm{VC}$ was added to the culture insert. Images were captured and analyzed at $0 \mathrm{~h}, 24 \mathrm{~h}$, and $48 \mathrm{~h}$ after wounding. The percent wound recovery was analyzed using Wimscratch Quantitative Wound Healing Image Analysis (Wimasis Gmbh, Germany).

\section{Western blot (WB) assay}

To detect the protein-level marker expression in hAECs and hGCs, cells were dissolved and dissociated in lysis buffer (Beyotime Biotechnology, China). After extracting the protein from the cells, $20 \mu \mathrm{g}$ of protein was loaded onto $10 \%$ gels and fractioned via $10 \%$ or $20 \%$ SDS-PAGE. Then, primary antibodies were used to bind the protein after the separated proteins were electroblotted onto PVDF. The expression of the proteins was tested by incubating with secondary antibodies and detected with enhanced chemiluminescence. By analyzing the gray value of the band of interest with ImageJ software (National Institutes of Health, USA), the expression levels of the proteins were calculated. Each experiment was repeated at least three times. The information of the first antibody is listed in Supplementary Table 2.

\section{Premature ovarian insufficiency (POI) mouse model establishment}

Female C57B6L/J mice aged 8 weeks were purchased from the Institute of Animal Research at Nanjing Medical University. The Ethics Committee of Nanjing Medical University (approval number: 20170480) approved this study. Mice were equally and randomly sorted into 4 groups: a normal group with no treatment (NG, $n=10$ ), a POI group treated with CTX followed by the transplantation of PBS (POI, $n=10$ ), an hAECs group treated with CTX following transplantation of hAECs (hAECs, $n=10$ ), and a VChAECs group treated with CTX following transplantation of the VC-treated hAECs. CTX was administered by intraperitoneal injection at a concentration of $120 \mathrm{mg} / \mathrm{kg}$.

\section{Cell transplantation}

Probe DiO (Invitrogen, USA, Cat: D275) was employed to trace VC-hAECs and hAECs after transplantation. After labeling with $\mathrm{DiO}$ according to the manufacturer's instructions, VC-hAECs and hAECs were dissolved in and washed with PBS before being resuspended in PBS at a volume of $10 \mu \mathrm{l}$. Two weeks after CTX injection, VC-hAECs and hAECs were transplanted into mouse ovaries through the caudal vein with microinjection needles by laparotomy. Mice in the POI group were injected with $10 \mu \mathrm{l}$ of PBS as a control.

\section{Immunohistochemical staining}

At 3, 7, and 14 days after cell transplantation, mice were sacrificed, and ovarian tissues were taken. Ovarian tissues were cut at a thickness of $5 \mu \mathrm{m}$ and then fixed in $4 \%(\mathrm{w} / \mathrm{v})$ paraformaldehyde (Sigma, USA). After being permeated with $0.1 \%$ Triton-X-100 for $5 \mathrm{~min}$ at $4{ }^{\circ} \mathrm{C}$, ovarian sections were blocked with $4 \%$ BSA (Sigma, USA) for $1 \mathrm{~h}$ at room temperature. After three washes, ovarian sections were incubated with primary antibodies overnight at $4{ }^{\circ} \mathrm{C}$. The primary antibodies against human-MVH (Abcam, USA, catalog number: ab13840) were used to label the ovarian cells. Next, mouse sections were washed with PBS three times after rewarming. A PE-conjugated secondary antibody was used to stain ovarian tissues by incubation in a dark room for $30 \mathrm{~min}$ at room temperature. Finally, ovarian sections were mounted using Hoechst-Fluoromount-G (Southern Biotech, USA) and viewed under a Nikon Eclipse E800 microscope.

\section{Alkaline phosphatase (AP) staining}

The expression of AP at the surface of hAECs and VChAECs was measured with an AP staining kit (System Biosciences, Canada) following the manufacturer's instructions. In brief, cells were washed with PBS before being fixed for $5 \mathrm{~min}$ at room temperature. Then, the fixing solution was removed, and the AP substrate solution was added to the culture plate. After incubation for $20 \mathrm{~min}$ at room temperature, staining solution was given to stop the reaction. Finally, the $\mathrm{AP}^{+}$cells (blue stained) were observed with a light microscope. 


\section{Hematoxylin and eosin (HE) staining and ovarian follicle count}

At 8 weeks posttransplantation, the mice were euthanized. Then, the ovaries on both sides of each mouse were removed and fixed in 10\% paraformaldehyde for 2 $\mathrm{h}$ at room temperature. Then, the ovaries were embedded in paraffin and sectioned at a thickness of $5 \mu \mathrm{m}$. Five sections were collected from each ovary. The ovarian structure and follicle phenotype are shown by staining with HE. Primordial follicles, primary follicles, secondary follicles, antral follicles, and total follicles were classified and calculated. To avoid recounting, each oocyte was counted once. This experiment was repeated at least three times.

\section{Cytokine array}

Paracrine profiles of hAECs and VC-hAECs were measured by protein antibody array (RayBiotech, USA). Culture media from hAECs and VC-hAECs was collected and centrifuged at $13,000 \mathrm{rpm}$ for $20 \mathrm{~min}$ at $2-8{ }^{\circ} \mathrm{C}$ before incubation with antibodies. Images were captured with an Axon GenePix laser scanner. Then, fluorescence intensity data were analyzed using RayBio Analysis Tool software.

\section{Enzyme-linked immunosorbent assay (ELISA)}

To detect hormone levels in mice, the protein levels of serum E2, FSH, and AMH in mice were measured. At $0,1,2,3,4$, and 5 weeks posttransplantation, blood samples $(0.5 \mathrm{ml})$ were collected. Then, the blood samples were centrifuged at $4000 \mathrm{r} / \mathrm{min}$ for $10 \mathrm{~min}$ and the blood cells were discarded. When detecting the paracrine activity of selected cytokines in hAECs and VC-hAECs, culture media was collected and centrifuged at $4000 \mathrm{r} / \mathrm{min}$ for 10 min. The supernatant was isolated and detected by ELISA kits (Mybiosource, USA) following the manufacturer's instructions. In brief, $50 \mu \mathrm{l}$ of serum or culture medium was added to one test well, followed by incubation and washing. After adding Stop Solution, samples were immediately measured by a spectrophotometer (Varian Company, Australia). Each sample was detected at least three times.

\section{Statistical analysis}

All experiments in this study were repeated at least 3 times. The values are shown as the mean \pm SD. Before Scheffe's $t$ test (SPSS 17.0 software) was conducted, oneway ANOVA was performed. Probability values $<5 \%$ were considered significant.

\section{Results}

\section{VC promoted the proliferation of hAECs}

First, the roles of VC and VPA (Valproic acid) in regulating the propagation and apoptosis of hAECs

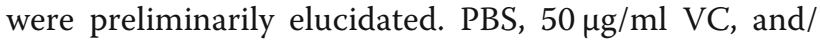
or $50 \mu \mathrm{g} / \mathrm{ml} \mathrm{VPA}$ were added to the culture media of hAECs at passage 5 every $24 \mathrm{~h}$. The light images and cell counting results in Fig. 1a show that the cell density of VC-treated hAECs was dramatically higher than that of PBS-treated hAECs 7 and 14 days after treatment. However, the cell densities of hAECs treated with only VPA and hAECs treated with both $\mathrm{VC}$ and VPA were lower than those of hAECs treated with PBS at 7 days. The FACS outcome 7 days after treatment verified these observations, and the percent $\mathrm{KI}^{+} 7^{+}$hAECs was significantly increased in VCtreated group compared with the PBS-treated group (Fig. 1b). The percent ANNEXIN $\mathrm{V}^{+}$hAECs significantly and dramatically increased after treatment with only VPA and treatment with both VC and VPA compared with hAECs treated with only PBS (Fig. 1c). In summary, our results indicated that $\mathrm{VC}$ promoted hAEC proliferation and VPA induced hAEC apoptosis.

\section{VC facilitated cell migration and extended the life span of hAECs}

Then, the optimal effective concentration of $\mathrm{VC}$ was evaluated by adding PBS, $25 \mu \mathrm{g} / \mathrm{ml} \mathrm{VC,} 50 \mu \mathrm{g} / \mathrm{ml} \mathrm{VC}$, or $100 \mu \mathrm{g} / \mathrm{ml} \mathrm{VC}$ to the culture media of hAECs. Wound healing assay results illustrated that cell migration was dramatically elevated in $50 \mu \mathrm{g} / \mathrm{ml} \mathrm{VC-}$ treated hAECs at $24 \mathrm{~h}$ and $48 \mathrm{~h}$ posttreatment (Fig. 2a). Moreover, trypan blue staining results showed that the percent viable hAECs at P10 was significantly and dramatically increased after administration of $50 \mu \mathrm{g} / \mathrm{ml} \mathrm{VC} \mathrm{(Fig.} \mathrm{2b).} \mathrm{By} \mathrm{analyzing} \mathrm{the}$ cell cycle of hAECs at P10, we found that the percentage of cells in the $S$ phase increased the most in the $50 \mu \mathrm{g} / \mathrm{ml} \mathrm{VC-treated} \mathrm{hAECs} \mathrm{(Fig.} \mathrm{2c).} \mathrm{The} \mathrm{WB}$ results also showed the highest expression level of telomerase marker (hTERT) in $50 \mu \mathrm{g} / \mathrm{ml} \mathrm{VC-treated}$ hAECs (Fig. 2d). Taken together, the proliferation, migration, and self-renewal of hAECs was greatly facilitated after $\mathrm{VC}$ treatment. Among the three doses of $\mathrm{VC}, 50 \mu \mathrm{g} / \mathrm{ml}$ showed the most effective outcomes.

\section{VC promoted pluripotency marker expression levels in hAECs}

To unveil the role of $\mathrm{VC}$ in regulating the pluripotency of hAECs, the expression of pluripotency markers in hAECs and hAECs treated with $50 \mu \mathrm{g} / \mathrm{ml}$ VC (VC-hAECs) was detected. According to our 


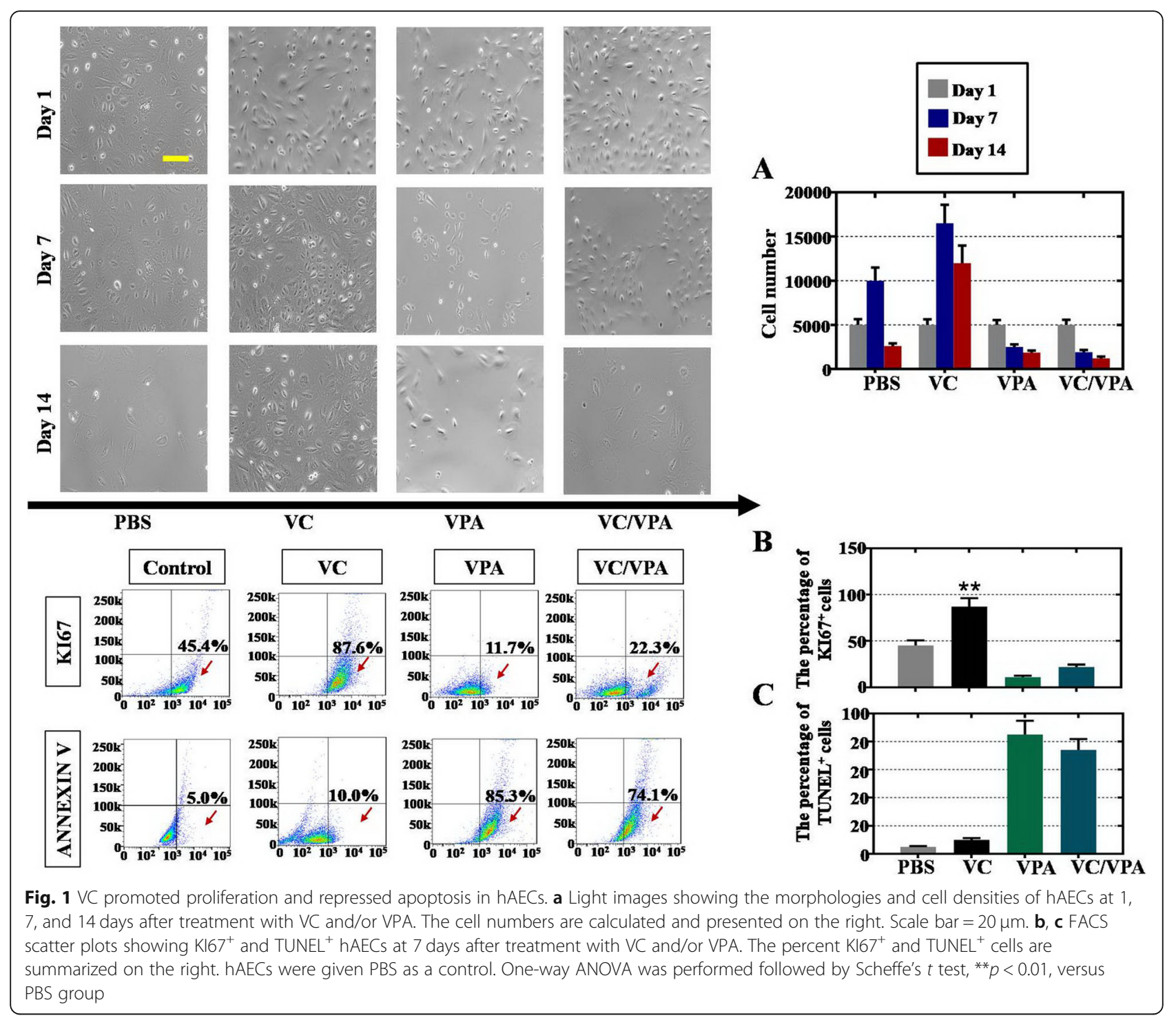

FACS results, $50 \mu \mathrm{g} / \mathrm{ml} \mathrm{VC}$ treatment greatly increased the percentages of OCT4-, NANOG-, SSEA4-, and TRA-1-81-positive cells in hAECs at P1 (increased from $4.99 \%, 7.34 \%, 11.1 \%$, and $8.52 \%$, respectively, to $81.6 \%, 92.6 \%, 97.9 \%$, and $76.8 \%$, respectively; Fig. 3a) and P5 (increased from 4.99\%, 7.34\%, 11.1\%, and $8.52 \%$, respectively, to $81.6 \%, 92.6 \%, 97.9 \%$, and $76.8 \%$, respectively; Fig. $3 \mathrm{~b})$. Using an enzymatic assay and a colorimetric method, our results showed that the expression of alkaline phosphatase (AP), a universal pluripotent marker for all types of pluripotent stem cells, was significantly elevated in VC-hAECs compared with hAECs (Fig. 3c). The WB results also demonstrated significantly upregulated expression of pluripotency markers (OCT4, NANOG, SSEA4, and TRA-1-81) in VC-hAECs compared with hAECs at P1 (Fig. 3d) and P5 (Fig. 3e). In addition, western blot assay was employed to test the protein level of ectoderm (Sox1, Nestin), mesoderm ( $T, C D 31)$, and endoderm (Sox17, AFP). Our results indicated that vitamin $\mathrm{C}$ improved the protein level of ectoderm, mesoderm, and endoderm more powerfully than without vitamin $C$ treatment (Supplementary Fig. 1). Altogether, our results demonstrated that VC promoted hAEC pluripotency.

Ovarian function was more powerfully rescued in the VChAEC-transplanted POI mouse model than in the hAECtransplanted $\mathrm{POI}$ mouse model

PBS, $2 \times 10^{5}$ VC-hAECs, or hAECs were injected into the ovaries of cyclophosphamide (CTX)-induced POI mice to further elucidate the therapeutic potential of VC-hAECs to treat POI. The therapeutic effects of VC-hAECs and hAECs were assessed by counting follicles and measuring hormone levels. Primordial, primary, secondary, and antral follicles can be observed 


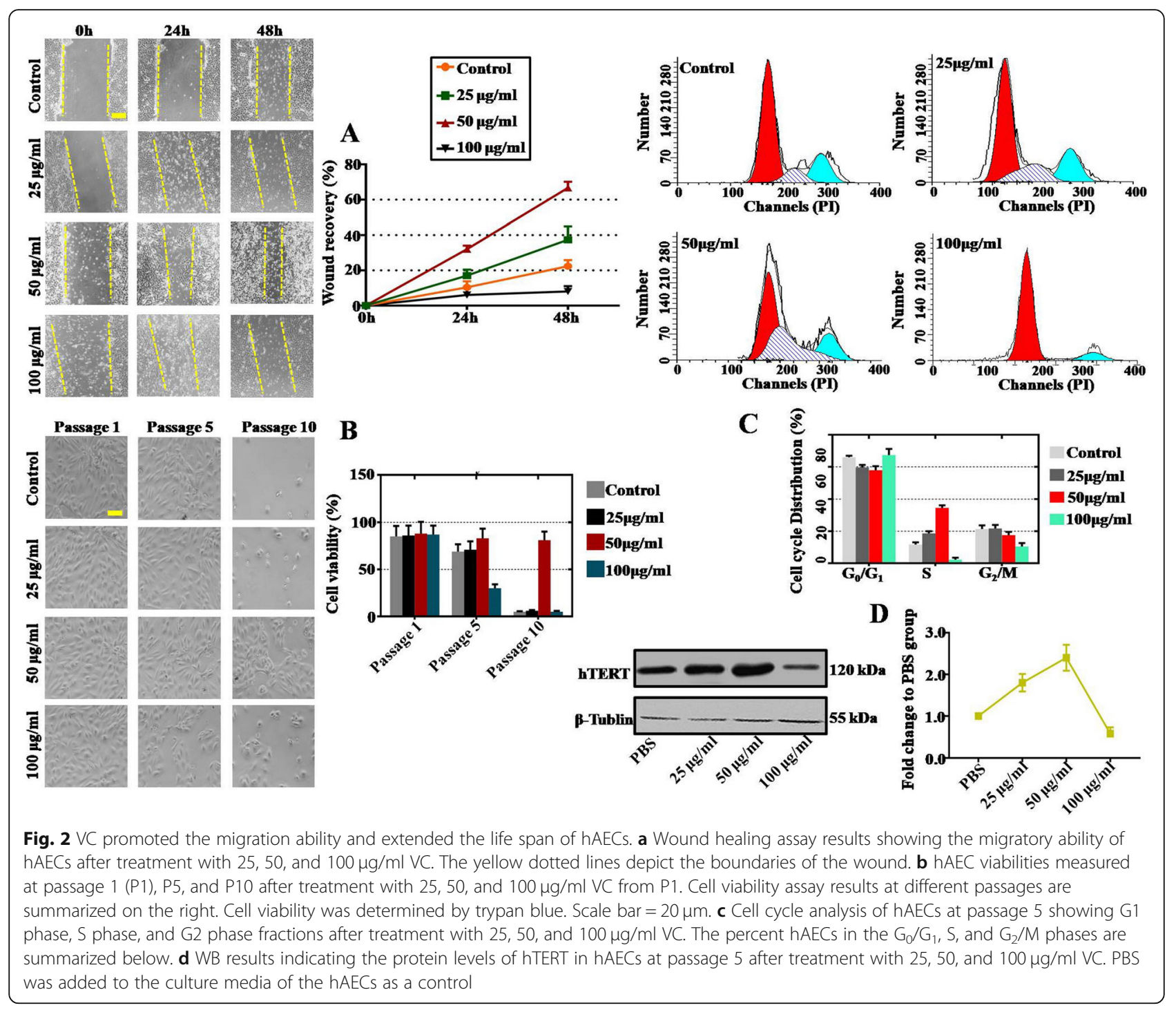

in the ovaries of VC-hAEC-transplanted POI mice (Fig. 4). Our data indicated that the numbers of antral and total follicles were significantly increased in the ovaries of VC-hAEC-transplanted POI mice compared with hAEC-transplanted POI mice and PBSinjected POI mice at 8 weeks posttransplantation (Fig. 4a, b). The serum hormone levels of E2 and $\mathrm{AMH}$ during the 8-week follow-up significantly and progressively increased in both hAEC- and VC-hAECtransplanted POI mice compared with the PBSinjected POI mice. Furthermore, the serum levels of $\mathrm{E} 2$ and $\mathrm{AMH}$ at 8 weeks posttransplantation were significantly higher in VC-hAEC-transplanted POI mice than in hAEC-transplanted POI mice (Fig. 4c, d). At 8 weeks postoperation, the serum level of FSH was significantly reduced in the VC-hAEC-transplanted group compared with the hAEC-transplanted group (Fig. 4e). Collectively, our data indicated that VC enhanced the therapeutic potential of hAECs in a POI mouse model.

\section{hGC marker expression levels were elevated after coculture with VC-hAECs}

A human ovarian granulosa cell line (GC1a) was donated by Dr. Hitoshi Okamura at Kumamoto University and treated with CTX to establish a POI model in vitro (POI group). After coculture with hAECs (hAECs/POI group) and VC-hAECs (VC-hAECs/POI group), the expression of hGC markers (FSHR, AMH, FOXL2, CYP19A1) was examined. As shown in Fig. $5 \mathrm{a}, \mathrm{b}$, the FACS results showed that the percent $\mathrm{FSHR}^{+} \mathrm{AMH}^{+}$and FOXL2 ${ }^{+} \mathrm{CYP} 19 \mathrm{~A}^{+}{ }^{+} \mathrm{hGCs}$ in the VC-hAECs/POI group $(85.9 \%$ and $91.5 \%$, respectively) were significantly higher than those of the hAECs/POI group (58.0\% and 59.0\%) and POI group (29.5\% and $26.6 \%)$. This result was further validated 


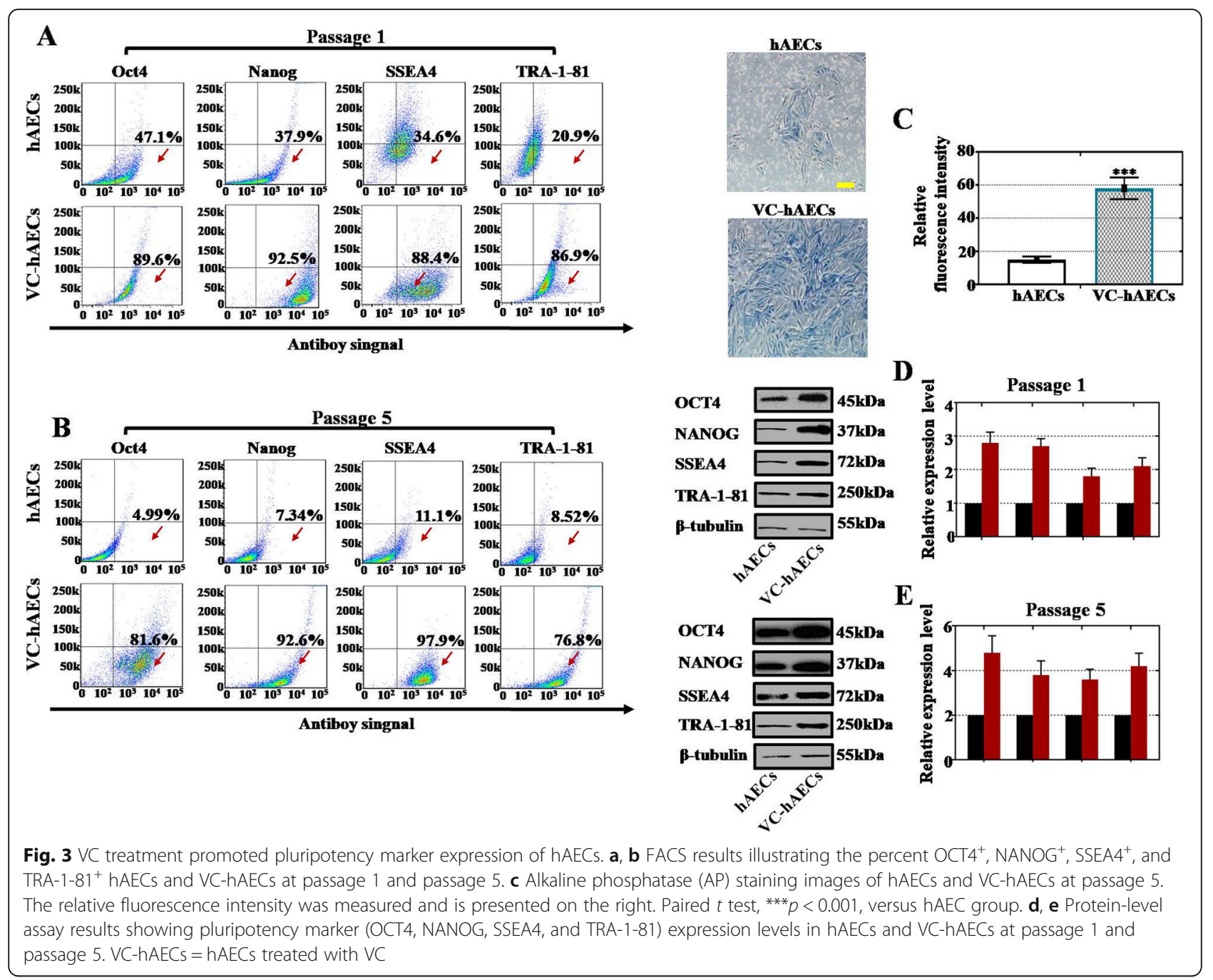

by WB analysis as shown in Fig. 5c: the protein levels of FSHR, AMH, FOXL2, and CYP19A1 were significantly elevated in the VC-hAECs/POI group (4-fold, 5-fold, 4-fold, and 4-fold increase compared with the POI group, respectively) when compared with the hAECs/POI group (2-fold, 2-fold, 2-fold, and 2-fold increase compared with the POI group, respectively). Therefore, $\mathrm{VC}$ was demonstrated to elevate the restorative effect of hAECs in treating CTX-damaged hGCs.

Ovarian marker expression levels were greater in the ovaries of the VC-hAEC-transplanted mice than in the hAEC-transplanted POI mice model

Then, ovarian transplantations of DiO-labeled VChAECs or hAECs were conducted in CTX-induced POI mice, followed by detection of the expression of ovarian markers (FSHR, AMH, FOXL2, CYP19A1) and analyzation of transplant survival. According to the FACS results, the percent $\mathrm{FSHR}^{+} \mathrm{AMH}^{+}$and FOXL2 ${ }^{+} \mathrm{CYP}_{19 \mathrm{~A} 1^{+}}$in ovarian cells in VC-hAECtransplanted POI mice (81.9\% and $92.9 \%$, respectively) were significantly greater than hAEC-transplanted POI mice $(48.2 \%$ and $47.7 \%)$ and POI mice $(25.0 \%$ and $30.2 \%$, Fig. $6 a, b)$. At 3,7 , and 14 days posttransplantation, POI mouse ovaries were sectioned and stained for the ovarian marker MVH. Immunofluorescence images at 3 days showed similar $\mathrm{DiO}$ intensities (green) in ovarian tissues between the groups. At 7 days posttransplantation, more DiO-labeled VChAECs were observed than DiO-labeled hAECs in ovarian tissues. At 14 days posttransplantation, the DiO-labeled hAECs were almost all cleared, while DiO-labeled VC-hAECs could still be observed (Fig. 6c). In summary, $\mathrm{VC}$ was demonstrated to enhance the transplantation survival of hAECs and promote the rescue effect of hAECs in preserving CTX-damaged ovaries. 


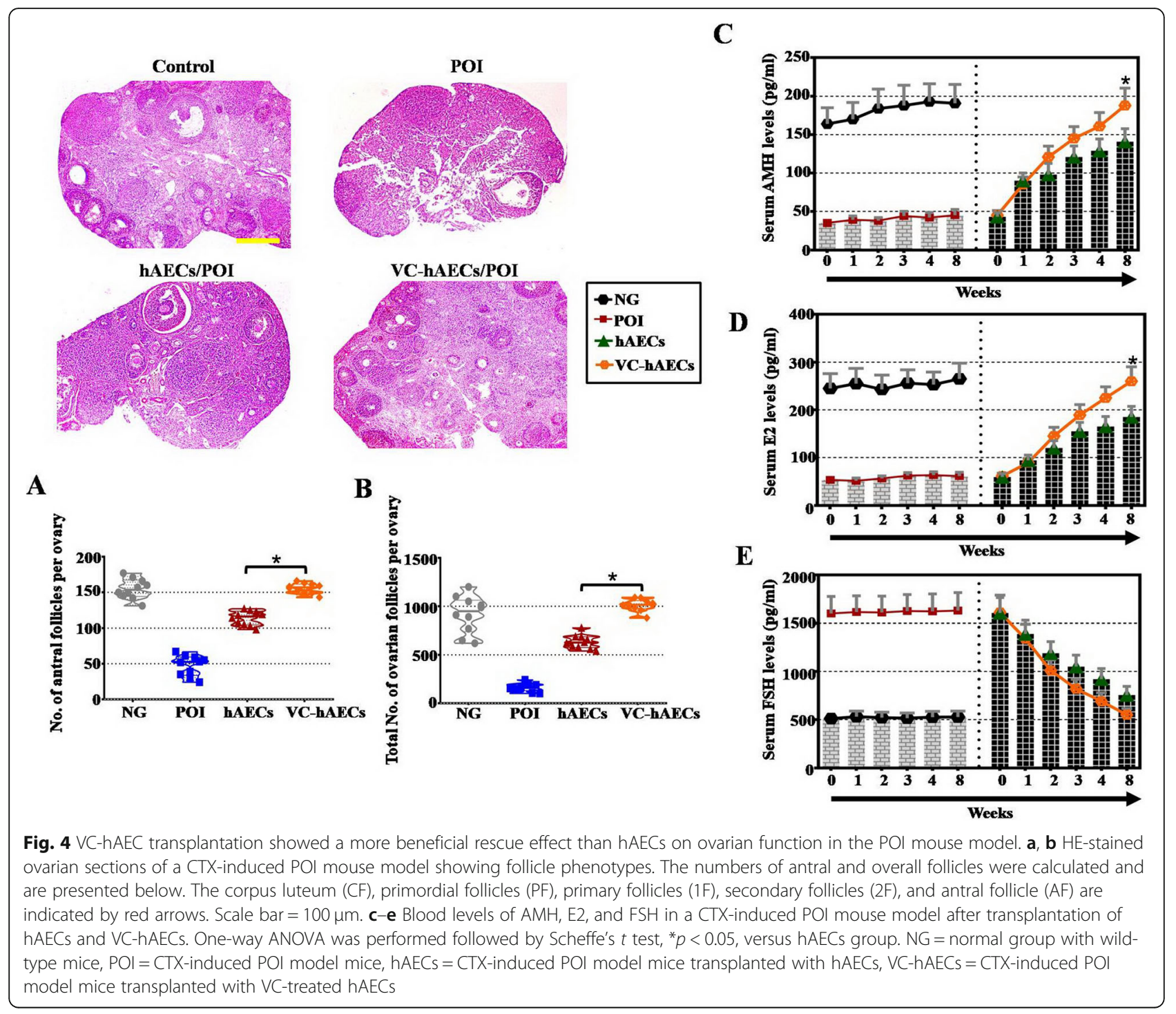

VC elevated the paracrine activity of hAECs

To unveil the paracrine function of cytokines in VChAECs, the culture media from VC-hAECs and hAECs were collected and underwent cytokine array analysis. The heatmap in Fig. 7a shows the cytokine profile of VC-hAECs and hAECs. The secretion of growth factors in hAECs was generally upregulated after VC treatment. The protein levels of secreted EGF, HGF, and bFGF from VC-hAECs were all over 10-fold higher than from the hAECs (Fig. 7b). ELISA results verified our previous findings and showed that the secreted protein levels of EGF, HGF, and bFGF from VC-hAECs were significantly increased compared with those from hAECs (Fig. 7c-e).

\section{Discussion}

The present study is the first to report that supplementation with $\mathrm{VC}$ in vitro could promote proliferation and suppress apoptosis in hAECs. However, VPA supplementation did the opposite (Fig. 1). In accordance with previous studies, VC treatment was demonstrated to increase the proliferation rate in various kinds of mesenchymal stem cells [29, 32]. VPA was reported to accelerate the proliferation and differentiation of neural stem cells but inhibit the propagation of mesenchymal stem cells and epithelial cells [33-35].

Migration and homing ability is critical for hAECbased therapy because these cells are expected to migrate to injury tissues [36]. Previous study demonstrated that the hAECs were found to migrate along nerve fibers in corpus callosum in the treatment of neurological diseases after transplantation [37]. Our results show that the migration ability of hAECs was highly facilitated by $50 \mu \mathrm{g} / \mathrm{ml} \mathrm{VC}$ 


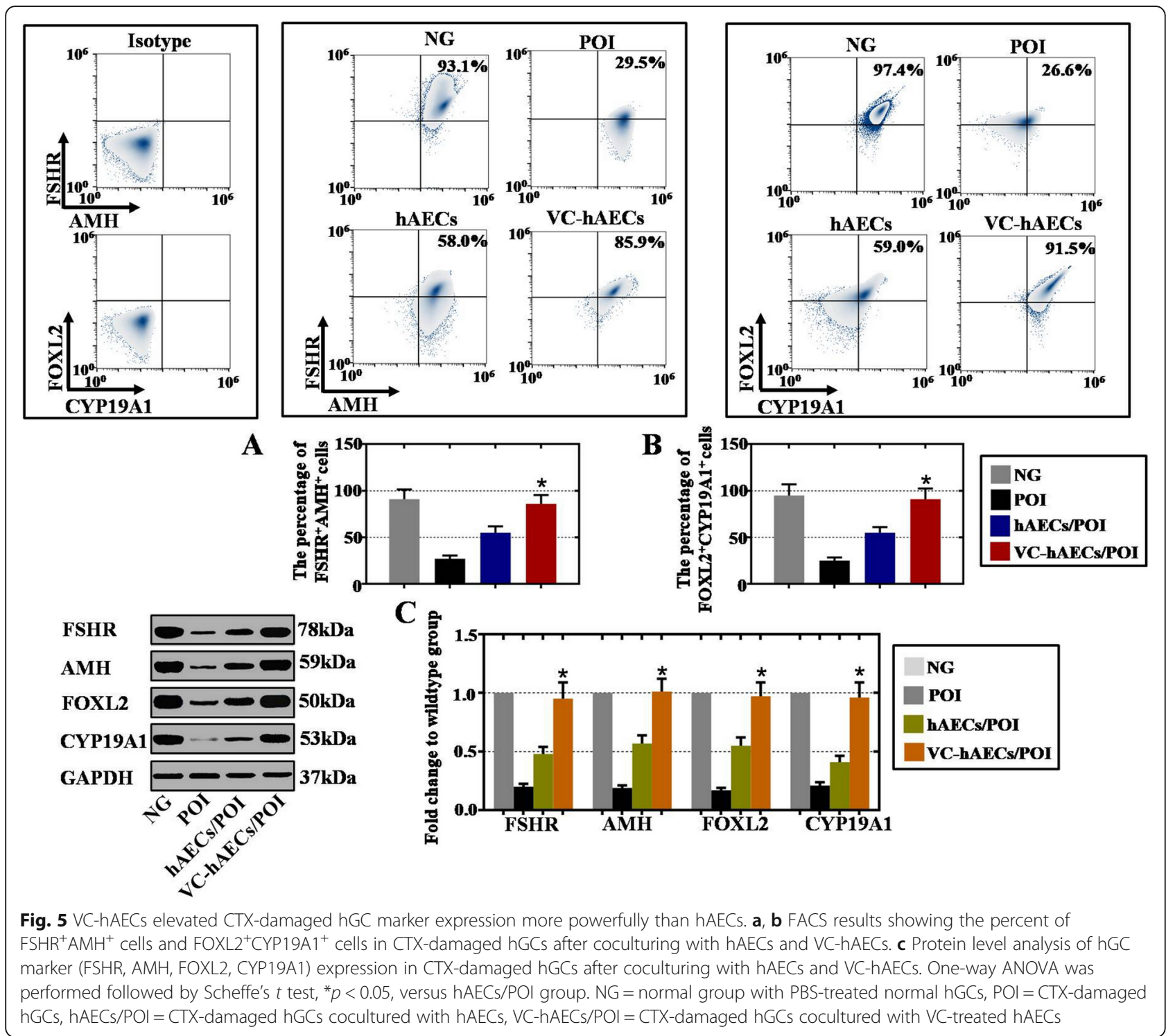

(Fig. 2a). Various factors influence the migration and homing efficiency in MSC, such as cell aging and culture conditions [36]. In our study, cell cycle

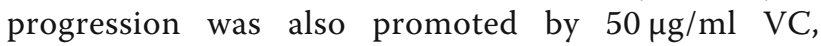
with an increased percentage in the $S$ phase (Fig. 2c). Previous studies indicated that VC promoted the cell cycle by upregulating cyclin E1 and CDK2 and downregulating p53 and p21 [32]. The life span and telomere expression of hAECs was extended by $50 \mu \mathrm{g} / \mathrm{ml} \mathrm{VC} \mathrm{(Fig.} \mathrm{2b,} \mathrm{d).} \mathrm{To} \mathrm{sum} \mathrm{up,} \mathrm{the} \mathrm{optimum}$ working concentration of $\mathrm{VC}$ was found to be $50 \mu \mathrm{g} / \mathrm{ml}$ (Fig. 2). This may be attributed to the antioxidant role of VC [38]. Consistent with our findings, VC blocked the aberrant self-renewal of human hematopoietic stem cells [39]. In addition, we found that $100 \mu \mathrm{g} / \mathrm{ml} \mathrm{VC}$ is toxic to hAECs.
Others have also suggested that a high dose of $\mathrm{VC}$ could inhibit cell proliferation [40-42].

The expression of pluripotency markers in hAECs was greatly increased after VC treatment (Fig. 3). Previous studies have highlighted the upregulated SOX2 and OCT4 expression after adding VC [28]. Here, we showed that expression of NANOG, SSEA4 and TRA-1-81 was also activated by VC. Researchers suggested that VC promoted pluripotency in induced pluripotent stem cell (iPSC) reprogramming by inhibiting p53 and p 21 expression [25] and restoring $\mathrm{H} 3$ lysine 4 trimethylation at Dlk1-Dio3 loci [43]. Others have indicated that VC induced a pluripotent state in embryonic stem cells by inducing microRNA-143 expression [23].

Finally, we observed a more powerful rescue effect of VC-treated hAECs (VC-hAECs) compared with 


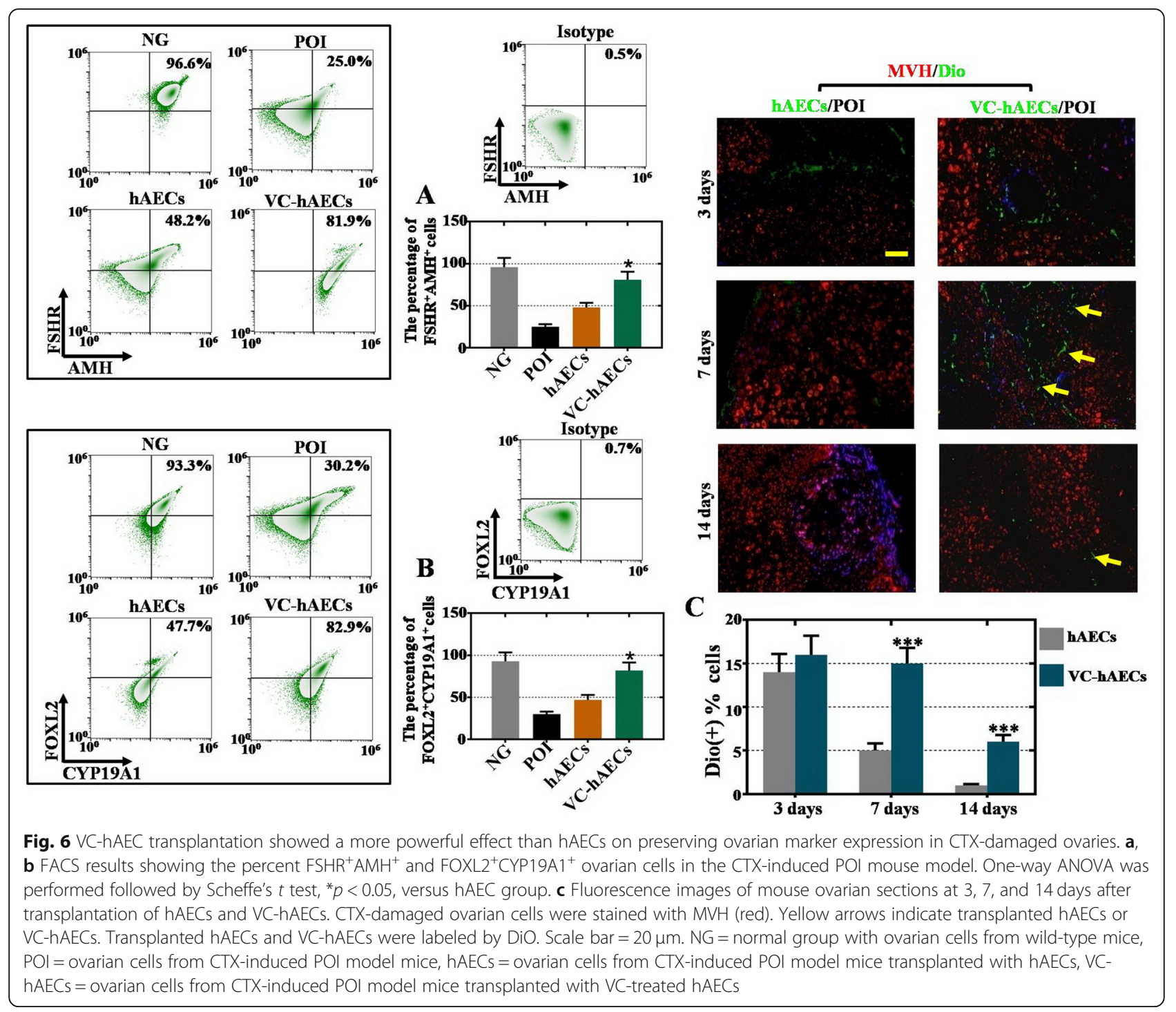

hAECs in treating POI disease (Figs. 4, 5, and 6). First, ovarian function was improved in VC-hAECtransplanted POI mice compared with the therapeutic effect of hAECs (Fig. 4). Second, after coculture with CTX-damaged hGCs, hGC marker expression was greater in the VC-hAEC group than in the hAEC group (Fig. 5). Third, in the ovaries of POI mice, ovarian markers were more highly expressed in the VC-hAEC transplanted group than in the hAEC transplanted group. Furthermore, transplanted VChAECs survived much longer than hAECs after ovary injection (Fig. 6). All these data elucidated the powerful role of $\mathrm{VC}$ in enhancing the therapeutic effects of hAECs. Whether VC-hAEC transplantation would achieve better outcomes than other graft resources (for example, hBMSCs, hADMSCs, or hUCMSCs) in cell-based therapy of POI needs further investigation.
By indirect coculture, VC-hAECs were found to more powerfully restore CTX-damaged hGCs compared with hAECs (Fig. 5). This result suggested that VC-hAECs showed therapeutic effects in a noncontact manner. In support of our findings, recent studies have suggested the paracrine mechanism of mesenchymal stem cell therapy [44]. Our previous study also demonstrated that hepatocyte growth factor (HGF) and epidermal growth factor (EGF) secreted from hAMSCs play a critical role in reversing ovarian aging [17]. In this study, VC-hAECs secreted more trophic factors than hAECs, including HGF and EGF (Fig. 7). Future studies will answer the question of whether the beneficial therapeutic effect of VC-hAECs is due to paracrine function or mediated by extracellular vesicles.

In conclusion, this study demonstrated that $\mathrm{VC}$ could increase the proliferation and migration ability 


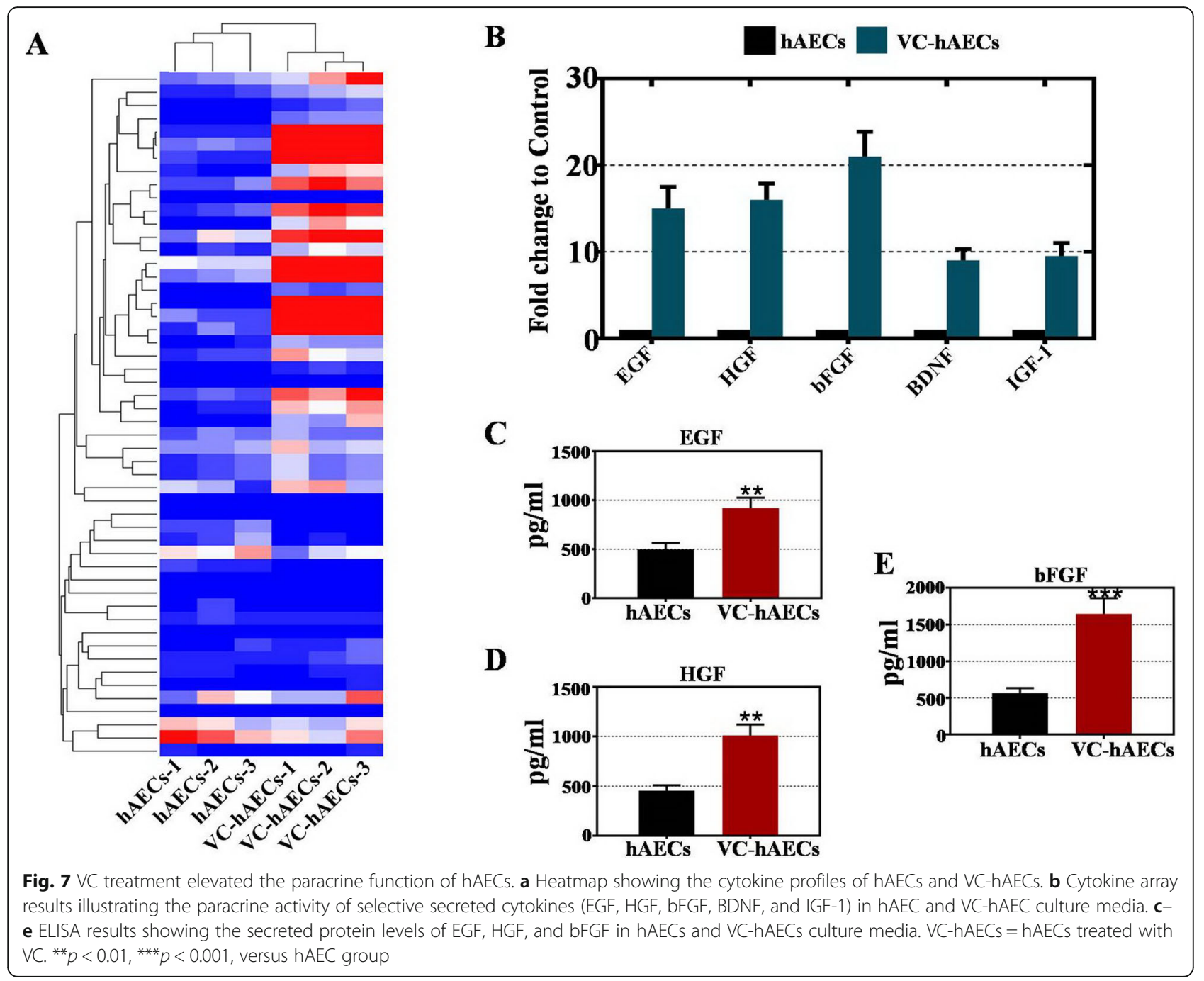

and promote pluripotency marker expression and paracrine function of hAECs in vitro. Furthermore, the therapeutic potential of hAECs in preserving ovarian cells and rescuing ovarian function was greatly increased after VC treatment. Our research shed light on transplant modification by small molecules and suggested VC-treated hAECs as a beneficial graft resource in treating POI.

\section{Supplementary information}

Supplementary information accompanies this paper at https://doi.org/10. 1186/s13287-020-01666-y.

Additional file 1: Figure S1. VC treatment improved differentiation potential of hAECs into three germ cell lineage. After VC treatment, the protein level of ectoderm (Sox1, Nestin), mesoderm (T, CD31), endoderm (Sox17, AFP) were elevated.

Additional file 2: Table S1. Information regarding the flow cytometry antibodies.

Additional file 3: Table S2. Information regarding the western blot antibodies.

\section{Abbreviations}

hAEC: Human amniotic epithelial cell; VC-hAEC: VC-treated human amniotic epithelial cell; hAMSC: Human amniotic mesenchymal stem cell; VC: Vitamin C; VPA: Valproic acid; hGC: Human ovarian granulosa cell; POF: Premature ovarian failure; hADSCs: Adipose-derived mesenchymal stem cells; hBMSCs: Human bone marrow mesenchymal stem cells; hUCMSCs: Human umbilical cord-derived mesenchymal stem cells; hTERT: Human telomerase reverse transcriptase; IVF: In vitro fertilization; CTX: Cyclophosphamide;

POI: Premature ovarian insufficiency; IPSC: Induced pluripotent stem cells; HGF: Hepatocyte growth factor; EGF: Epidermal growth factor; AP: Alkaline phosphatase

\section{Authors' contributions}

S.H. and C.D. performed the cellular modify by VC and stem cell therapeutic assays in vivo and in vitro. H.S. participated in the statistical analysis and composed the figures in the manuscript. hGC collection and purification were completed by Q.Z.. J.L. performed the partial immunofluorescence experiments. C.Q. participated in the mice feeding and carried out the partial HE assays. B.H., J.T., and H.L. planned the all experiments and drafted the manuscript. All the authors read and approved the final manuscript.

\section{Funding}

Cellular samples, experimental animal, and sectional experiment reagents were supported by the grants from the National Natural Science Foundation of China (81801515, 81801494, 81801478); partial experiment reagents were acquired by Suzhou introduce expert team of clinical medicine 
(SZYJTD201708, SZYJTD201707) and Suzhou talent training program (GSWS2019005); and the Suzhou Key Medical Center (SZZXX201505) supported the salary of the postdoctors in this paper.

\section{Availability of data and materials}

All the data generated or analyzed during this study are included in this published article.

\section{Ethics approval and consent to participate}

The use of human ovarian granular cells was in accordance with the relevant guidelines and regulations, and the experimental protocols were approved by the Medical Ethics Committee of the Suzhou Hospital Affiliated to Nanjing Medical University (NJMU-2015-014). All the healthy donors provided written informed consent prior to participation in this study. Our investigation using experimental animals was conducted on the basis of the Nanjing Medical University Animal Center's specific guidelines and standards.

\section{Consent for publication}

Not applicable

\section{Competing interests}

The authors declare no conflicts of interest.

\section{Author details}

'Department of Obstetrics and Gynecology, Affiliated Suzhou Hospital of Nanjing Medical University, Suzhou Municipal Hospital, Suzhou 215002, China. ${ }^{2}$ Center of Reproduction and Genetics, Affiliated Suzhou Hospital of Nanjing Medical University, Suzhou Municipal Hospital, Suzhou 215002, China. ${ }^{3}$ State Key Laboratory of Reproductive Medicine, Nanjing Medical University, Nanjing 210029, China. ${ }^{4}$ Reproductive Medical Center of Gynecology and Obstetrics Department, Shengjing Hospital of China Medical University, Shenyang 110000, China. ${ }^{5}$ Key Laboratory of Reproductive Dysfunction Diseases and Fertility Remodeling of Liaoning Province, Shenyang 110000, China.

\section{Received: 10 December 2019 Revised: 12 March 2020}

Accepted: 30 March 2020 Published online: 22 April 2020

\section{References}

1. Kokcu A. Premature ovarian failure from current perspective. Gynecol Endocrinol. 2010;268:555-62 (2010).

2. Fu X, He Y, Xie C, Liu W. Bone marrow mesenchymal stem cell transplantation improves ovarian function and structure in rats with chemotherapy-induced ovarian damage. Cytotherapy. 2008;104:353-63 (2008).

3. Ding C, Zou Q, Wu Y, Lu J, Qian C, Li H, Huang B. EGF released from human placental mesenchymal stem cells improves premature ovarian insufficiency via NRF2/HO-1 activation. Aging. 2020;12:2992-3009 (2020).

4. Yang M, Lin L, Sha C, Li T, Zhao D, Wei H, Chen Q, Liu Y, Chen X, Xu W, Li Y, Zhu X. Bone marrow mesenchymal stem cell-derived exosomal miR-144-5p improves rat ovarian function after chemotherapy-induced ovarian failure by targeting PTEN. Lab Investig. 2019;19:19 (2019).

5. Yang Z, Du X, Wang C, Zhang J, Liu C, Li Y, Jiang H. Therapeutic effects of human umbilical cord mesenchymal stem cell-derived microvesicles on premature ovarian insufficiency in mice. Stem Cell Res Ther. 2019;101:250 (2019).

6. Huang B, Lu J, Ding C, Zou Q, Wang W, Li H. Exosomes derived from human adipose mesenchymal stem cells improve ovary function of premature ovarian insufficiency by targeting SMAD. Stem Cell Res Ther. 2018:91:216 (2018).

7. Waldron PV, Di Marco F, Kruczek K, Ribeiro J, Graca AB, Hippert C, Aghaizu ND, Kalargyrou AA, Barber AC, Grimaldi G, Duran Y, Blackford SJ, Kloc M, Goh D, Zabala Aldunate E, Sampson RD, Bainbridge JWB, Smith AJ, Gonzalez-Cordero A, Sowden JC, Ali RR, Pearson RA. Transplanted donor- or stem cell-derived cone photoreceptors can both integrate and undergo material transfer in an environment-dependent manner. Stem Cell Reports. 2018;102:406-21 (2018).

8. Pearson RA, Gonzalez-Cordero A, West EL, Ribeiro JR, Aghaizu N, Goh D, Sampson RD, Georgiadis A, Waldron PV, Duran Y, Naeem A, Kloc M, Cristante E, Kruczek K, Warre-Cornish K, Sowden JC, Smith AJ, Ali RR. Donor and host photoreceptors engage in material transfer following transplantation of post-mitotic photoreceptor precursors. Nat Commun. 2016;7:13029 (2016).

9. Huang B, Qian C, Ding C, Meng Q, Zou Q, Li H. Fetal liver mesenchymal stem cells restore ovarian function in premature ovarian insufficiency by targeting MT1. Stem Cell Res Ther. 2019;10(1):362 (2019).

10. Gupta S, Lodha P, Karthick MS, Tandulwadkar SR. Role of autologous bone marrow-derived stem cell therapy for follicular recruitment in premature ovarian insufficiency: review of literature and a case report of world's first baby with ovarian autologous stem cell therapy in a perimenopausal woman of age 45 year. J Hum Reprod Sci. 2018;112:125-30 (2018).

11. Yang Y, Lei L, Wang S, Sheng X, Yan G, Xu L, Liu J, Liu M, Zhen X, Ding L, Sun $H$. Transplantation of umbilical cord-derived mesenchymal stem cells on a collagen scaffold improves ovarian function in a premature ovarian failure model of mice. In vitro Cell Dev Biol Anim. 2019;554:302-11 (2019).

12. Sun L, Li D, Song K, Wei J, Yao S, Li Z, Su X, Ju X, Chao L, Deng X, Kong B, Li L. Exosomes derived from human umbilical cord mesenchymal stem cells protect against cisplatin-induced ovarian granulosa cell stress and apoptosis in vitro. Sci Rep. 2017;71:2552 (2017).

13. Zhang Q, Bu S, Sun J, Xu M, Yao X, He K, Lai D. Paracrine effects of human amniotic epithelial cells protect against chemotherapy-induced ovarian damage. Stem Cell Res Ther. 2017:81:270 (2017).

14. Zhang Q, Xu M, Yao X, Li T, Wang Q, Lai D. Human amniotic epithelial cells inhibit granulosa cell apoptosis induced by chemotherapy and restore the fertility. Stem Cell Res Ther. 2015;6:152 (2015).

15. Xiao GY, Cheng CC, Chiang YS, Cheng WT, Liu IH, Wu SC. Exosomal miR-10a derived from amniotic fluid stem cells preserves ovarian follicles after chemotherapy. Sci Rep. 2016;6:23120 (2016).

16. Ding C, Li H, Wang Y, Wang F, Wu H, Chen R, LV J, Wang W, Huang B. Different therapeutic effects of cells derived from human amniotic membrane on premature ovarian aging depend on distinct cellular biological characteristics. Stem Cell Res Ther. 2017;81:173 (2017).

17. Ding C, Zou Q, Wang F, Wu H, Chen R, Lv J, Ling M, Sun J, Wang W, Li H, Huang B. Human amniotic mesenchymal stem cells improve ovarian function in natural aging through secreting hepatocyte growth factor and epidermal growth factor. Stem Cell Res Ther. 2018;91:55 (2018).

18. Araujo AB, Salton GD, Furlan JM, Schneider N, Angeli MH, Laureano AM, Silla L, Passos EP, Paz AH. Comparison of human mesenchymal stromal cells from four neonatal tissues: Amniotic membrane, chorionic membrane, placental decidua and umbilical cord. Cytotherapy. 2017;195:577-85 (2017).

19. Toda A, Okabe M, Yoshida T, Nikaido T. The potential of amniotic membrane/amnion-derived cells for regeneration of various tissues. J Pharmacol Sci. 2007;1053:215-28 (2007).

20. Hwang BW, Kim SJ, Park KM, Kim H, Yeom J, Yang J-A, Jeong H, Jung H, Kim K, Sung YC, Hahn SK. Genetically engineered mesenchymal stem cell therapy using self-assembling supramolecular hydrogels. J Control Release. 2015;220(SupplementPA):119-29 (2015)

21. Partridge K, Yang X, Clarke NM, Okubo Y, Bessho K, Sebald W, Howdle SM, Shakesheff KM, Oreffo RO. Adenoviral BMP-2 gene transfer in mesenchymal stem cells: in vitro and in vivo bone formation on biodegradable polymer scaffolds. Biochem Biophys Res Commun. 2002;2921:144-52 (2002).

22. Brower JBS, Blumberg SMD, Carroll EBS, Pastar IP, Brem HMD, Chen WPR. Mesenchymal stem cell therapy and delivery systems in nonhealing wounds. Adv Skin Wound Care. 2011;24(11):524-32 2011.

23. Gao Y, Han Z, Li Q, Wu Y, Shi X, Ai Z, Du J, Li W, Guo Z, Zhang Y. Vitamin C induces a pluripotent state in mouse embryonic stem cells by modulating microRNA expression. FEBS J. 2015;2824:685-99 (2015).

24. Gao Y, Yang L, Chen L, Wang X, Wu H, Ai Z, Du J, Liu Y, Shi X, Wu Y, Guo Z, Zhang $Y$. Vitamin $C$ facilitates pluripotent stem cell maintenance by promoting pluripotency gene transcription. Biochimie. 2013;9511:2107-13 (2013).

25. Esteban MA, Wang T, Qin B, Yang J, Qin D, Cai J, Li W, Weng Z, Chen J, Ni S, Chen K, Li Y, Liu X, Xu J, Zhang S, Li F, He W, Labuda K, Song Y, Peterbauer A, Wolbank S, Redl H, Zhong M, Cai D, Zeng L, Pei D. Vitamin C enhances the generation of mouse and human induced pluripotent stem cells. Cell Stem Cell. 2010;61:71-9 (2010).

26. Eid W, Abdel-Rehim W. Vitamin C promotes pluripotency of human induced pluripotent stem cells via the histone demethylase JARID1A. Biol Chem. 2016;39711:1205-13 (2016).

27. Blaschke K, Ebata KT, Karimi MM, Zepeda-Martinez JA, Goyal P, Mahapatra S, Tam A, Laird DJ, Hirst M, Rao A, Lorincz MC, Ramalho-Santos M. Vitamin C induces Tet-dependent DNA demethylation and a blastocyst-like state in ES cells. Nature. 2013;5007461:222-6 (2013). 
28. Potdar PD, D'Souza SB. Ascorbic acid induces in vitro proliferation of human subcutaneous adipose tissue derived mesenchymal stem cells with upregulation of embryonic stem cell pluripotency markers Oct4 and SOX 2. Human Cell. 2010:234:152-5 (2010).

29. Choi KM, Seo YK, Yoon HH, Song KY, Kwon SY, Lee HS, Park JK. Effect of ascorbic acid on bone marrow-derived mesenchymal stem cell proliferation and differentiation. J Biosci Bioeng. 2008;1056:586-94 (2008).

30. Wang Y, Singh A, Xu P, Pindrus MA, Blasioli DJ, Kaplan DL. Expansion and osteogenic differentiation of bone marrow-derived mesenchymal stem cells on a vitamin C functionalized polymer. Biomaterials. 2006;2717:3265-73 (2006).

31. Yao ZY, Liu YM, Chen YL, Chen L, He SR, Zhang ZS. Ascorbic acid influences on extracellular matrix and structure of rabbit bone marrow mesenchymal stem cells. J Clin Rehabil Tissue Eng Res. 2018;229:1325-31 (2018).

32. Zhang P, Li J, Qi Y, Zou Y, Liu L, Tang X, Duan J, Liu H, Zeng G. Vitamin C promotes the proliferation of human adipose-derived stem cells via p53p21 pathway. Organogenesis. 2016;123:143-51 (2016).

33. Nan G, Li M, Liao W, Qin J, Cao Y, Lu Y. Effect of valproic acid on endogenous neural stem cell proliferation in a rat model of spinal cord injury [white star]. Neural Regen Res. 2009;47:513-7 (2009).

34. Lee HBS, Lee JYBS, Ha D-HBS, Jeong J-HP, Park J-BDDSMSDP. Effects of valproic acid on morphology, proliferation, and differentiation of mesenchymal stem cells derived from human gingival tissue. Implant Dent. 2018;271:33-42 (2018)

35. Kothary PC, Rossi B, Del Monte MA. Valproic acid induced human retinal pigment epithelial cell death as well as its survival after hydrogen peroxide damage is mediated by P38 kinase. Adv Exp Med Biol. 2015;854:765-72 (2015).

36. Sohni A, Verfaillie CM. Mesenchymal stem cells migration homing and tracking. Stem Cells Int. 2013;2013:130763 (2013).

37. Wu Z, Hui G, Lu Y, Liu T, Huang Q, Guo L. Human amniotic epithelial cells express specific markers of nerve cells and migrate along the nerve fibers in the corpus callosum. Neural Regen Res. 2012;71:41-5 (2012).

38. Cimmino L, Neel BG, Aifantis I. Vitamin C in stem cell reprogramming and cancer. Trends Cell Biol. 2018;289:698-708 (2018).

39. Cimmino L, Dolgalev I, Wang Y, Yoshimi A, Martin GH, Wang J, Ng V, Xia B, Witkowski MT, Mitchell-Flack M, Grillo I, Bakogianni S, Ndiaye-Lobry D, Martin MT, Guillamot M, Banh RS, Xu M, Figueroa ME, Dickins RA, AbdelWahab O, Park CY, Tsirigos A, Neel BG, Aifantis I. Restoration of TET2 function blocks aberrant self-renewal and leukemia progression. Cell. 2017; 1706:1079-1095.e1020 (2017).

40. Heckelen A, Hermel M, Kondring B, Schrage NF. Ascorbic acid reversibly inhibits proliferation of retinal pigment epithelial cells. Acta Ophthalmol Scand. 2004:825:564-8 (2004).

41. Lee SK, Kang JS, Jung DJ, Hur DY, Kim JE, Hahm E, Bae S, Kim HW, Kim D, Cho BJ, Cho D, Shin DH, Hwang YI, Lee WJ. Vitamin C suppresses proliferation of the human melanoma cell SK-MEL-2 through the inhibition of cyclooxygenase-2 (COX-2) expression and the modulation of insulin-like growth factor II (IGF-II) production. J Cell Physiol. 2008;2161:180-8 (2008).

42. Kim KN, Pie JE, Park JH, Park YH, Kim HW, Kim MK. Retinoic acid and ascorbic acid act synergistically in inhibiting human breast cancer cell proliferation. J Nutr Biochem. 2006;177:454-62 (2006).

43. Stadtfeld M, Apostolou E, Ferrari F, Choi J, Walsh RM, Chen T, Ooi SS, Kim SY, Bestor TH, Shioda T, Park PJ, Hochedlinger K. Ascorbic acid prevents loss of Dlk1-Dio3 imprinting and facilitates generation of all-iPS cell mice from terminally differentiated B cells. Nat Genet. 2012;444:398-405 S391-392 (2012).

44. Liang $X$, Ding $Y$, Zhang $Y$, Tse HF, Lian Q. Paracrine mechanisms of mesenchymal stem cell-based therapy: current status and perspectives. Cell Transplant. 2014;239:1045-59 (2014).

\section{Publisher's Note}

Springer Nature remains neutral with regard to jurisdictional claims in published maps and institutional affiliations.

Ready to submit your research? Choose BMC and benefit from:

- fast, convenient online submission

- thorough peer review by experienced researchers in your field

- rapid publication on acceptance

- support for research data, including large and complex data types

- gold Open Access which fosters wider collaboration and increased citations

- maximum visibility for your research: over $100 \mathrm{M}$ website views per year

At BMC, research is always in progress.

Learn more biomedcentral.com/submissions 